\title{
Misaligned from birth
}

Nature https://doi.org/10.1038/s41586-018-0819-2

(2018)

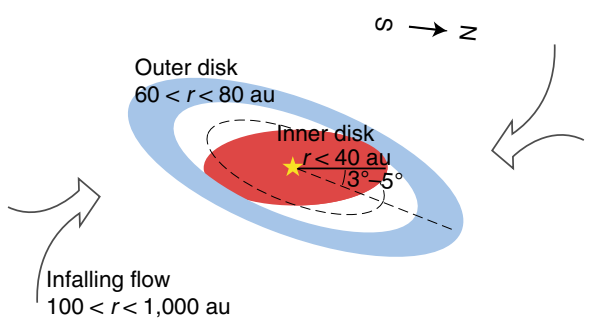

Credit: Springer Nature Ltd

In our Solar System, most of the planets orbit the Sun in the same plane, to within a few degrees. But for the Neptune-mass exoplanet GJ 436b, its orbital plane is perpendicular to the equator of its parent star. Are orbital planes misaligned at birth or did they evolve to be that way? Nami Sakai and co-workers use the latest ALMA observations of the disk system around a young protostar, IRAS 04368+ 2557 , to argue that planets can be formed from warped disks during formation.

In terms of stellar evolution, IRAS $04368+2557$ is at the earliest stage, class 0 , where rotating gas from the protostellar envelope is collapsing onto the disk and protostar. In fact, the system has an inner disk of radius $40 \mathrm{au}$ and an outer ring from 60 au out to $80 \mathrm{au}$. The two disks have orbital planes that differ by $3-5^{\circ}$ (the inner disk is shown in red in the figure; outer in blue). That such warping exists at class 0 suggests that previously observed warped structures began early in disk formation, thus explaining the diversity of orbital planes. Sakai et al. speculate that the warping could arise from changes in the angular momentum of the envelope gas due to asymmetries in the gas distribution in the parent stellar core (L1527); these lead to differences in infall rates (shown as arrows). Alternatively, the disk and envelope system could be misaligned with the magnetic field. Future observations will help discriminate between these ideas.

\section{May Chiao}

Published online: 8 January 2019

https://doi.org/10.1038/s41550-018-0681-z 